\title{
Lymphocytic mastopathy associated with infiltrating lobular breast carcinoma
}

\author{
R Chetty, A E Butler
}

\begin{abstract}
A case of lymphocytic mastopathy (LM) and concomitant infiltrating lobular breast carcinoma is described. The inflammatory infiltrate cuffed areas of carcinoma. The $L M$ changes also affected lobules that had not been infiltrated by the carcinoma, although adjacent areas contained both in situ and infiltrating tumour. A minor portion of the inflammation was distributed throughout the ducts.

The striking lobulocentricity of the inflammatory infiltrate corresponds to previous reports of $L M$, and distinguishes this case from the inflammatory response commonly associated with breast carcinomas. It is highly likely that the breast carcinoma induced the $L M$ changes in lobules not yet harbouring tumour.
\end{abstract}

(F Clin Pathol 1993;46:376-377)

Lymphocytic mastopathy (LM) and sclerosing lymphocytic lobulitis have been the subject of recent publications. ${ }^{1-3}$ The microscopic similarities with myoepithelial sialadenitis (MESA) and Hashimoto's thyroiditis have been highlighted, together with a putative autoimmune aetiology. ${ }^{1}$ The coexistence of LM with other autoimmune diseases (type 1 diabetes mellitus and Hashimoto's thyroiditis) has been noted and an autoimmune role inferred. ${ }^{3}$ Lymphoid infiltrates are common in breast carcinomas, ${ }^{4}$ but as Schwartz and Strauchen noted, this inflammatory infiltrate does not have the characteristic lobulocentricity of LM. ${ }^{1}$

\section{Pathology \\ Department, the Bendigo Hospital, Bendigo, Australia R Chetty \\ Department of Pathology and Laboratory Medicine, Mayo Clinic, Rochester, USA A E Butler \\ Correspondence to: Dr R Chetty, Department of Anatomical Pathology, The Royal Melbourne Hospital Grattan Street, Parkville, Melbourne, Victoria 3050, Australia \\ Accepted for publication 12 November 1992}

lowed by a total mastectomy and axillary clearance. She also received a course of radiotherapy. Nine months after the initial procedure, the patient remained well with no evidence of recurrence.

\begin{abstract}
Methods
All specimens were fixed in $10 \%$ buffered formalin and routinely processed. Haematoxylin and eosin, periodic acid Schiff (PAS) with and without diastase predigestion, and mucicarmine stains were used.

Immunohistochemical staining was performed on paraffin wax embedded tissue, using the peroxidase-antiperoxidase technique for the following: epithelial membrane antigen (EMA), L26 (CD 20, for B lymphocytes), UCHL1 (CD 45RO, for T lymphocytes), $\kappa$ and $\lambda$ light chains. All antibodies were obtained from Dakopatts (Santa Barbara, California, USA), and standard dilutions were used.
\end{abstract}

\section{Results}

Sections from the initial lumpectomy and the mastectomy specimens showed essentially the same findings. Features typical of a lobular carcinoma were evident: single file and "targetoid" patterns of infiltration by a relatively uniform population of cells (fig 1). More cohesive sheets of cells were also present and these were surrounded by lymphocytes. Areas containing foci of lobular carcinoma in situ were not attended by the lymphoid response. Several unaffected lobules were cuffed by a dense lymphoid infiltrate. In addition to this distinct lobulocentricity, the lobular epithelium was moderately attenuated and lymphocytes permeating the epithelium in the same manner as "lympho-epithelial lesions", were also observed (fig 2). Some of the lymphoid aggregates contained germinal centres (fig 2). Histiocytes and plasma cells formed a minor part of the inflammatory cell population. According to the grading scheme adopted by Schwartz and Strauchen, ${ }^{1}$ the severity of the lymphoid infiltrate corresponded to $3+$. The mastectomy specimen showed a milder form of $\mathrm{LM}$ and the infiltrate contained a large number of mast cells, which was probably related to the surgery performed 10 days earlier.

Immunohistochemical staining showed a preponderance of $B$ lymphocytes and a smaller population of scattered $\mathrm{T}$ lymphocytes. The B lymphocytes expressed both $\kappa$ and $\lambda$ light chains. The epithelial markers accentuated the lobular atrophy and attenuation caused by the lymphocytes. 


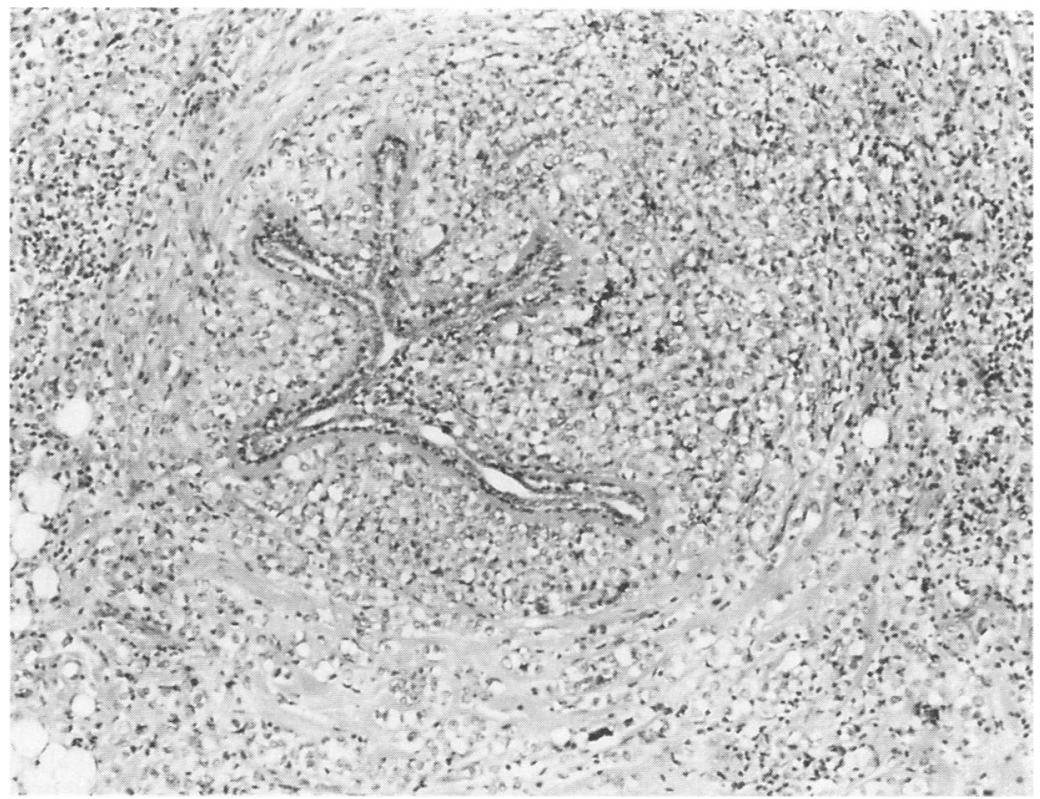

Figure 1 Uniform cells arranged in single file and targeted patterns typifying a classic lobular carcinoma (haematoxylin and eosin).

\begin{abstract}
Discussion
Lymphoid aggregates are fairly common in many breast carcinomas of no special type, as well as the histologically characteristic medullary carcinoma of the breast. ${ }^{5}$ In the case presented here, the infiltrating tumour was associated with a lymphoid infiltrate and lobules unaffected by the tumour were also surrounded by a similar infiltrate in an LM manner. The lack of a positive autoimmune profile at the time of presentation does not preclude the development of autoantibodies at a later stage. Furthermore, HLA-DR expression was not sought in our patient.
\end{abstract}

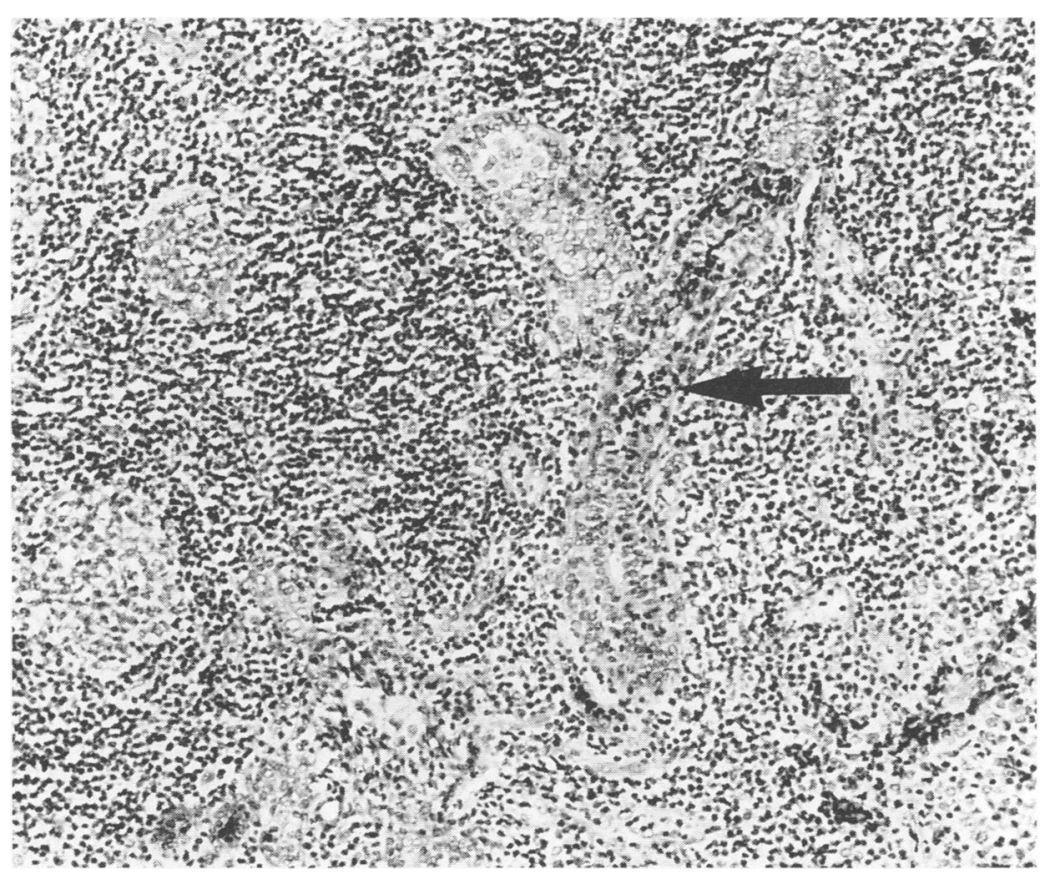

The histological similarity with myoepithelial sialadenitis and Hashimoto's thyroiditis is striking. Both these conditions are characterised by lymphoid aggregates and an autoimmune aetiology. They also contain socalled lymphoepithelial lesions.

The current case also showed focal permeation of epithelium by lymphocytes, though $\mathrm{LM}$ does not seem to be associated with the extensive destruction that is seen in benign lymphoepithelial lesions of the salivary glands or Hashimoto's thyroiditis. Distinction of benign lymphoepithelial lesions from lymphomas belonging to the category of mucosaassociated lymphoid tissue (MALT) lymphomas rests on the immunophenotypic or immunogenotypic demonstration of a monoclonal population of lymphoid cells. The infiltrate in this case cannot be separated from lymphoma on morphological grounds alone. Even with the lack of immunophenotypic demonstration of monoclonality, lymphoma cannot be discounted because immunoglobulin-gene rearrangement has been shown in benign lymphoepithelial lesions. ${ }^{6}$ Interestingly, several primary breast lymphomas morphologically resemble lymphomas arising from MALT. ${ }^{7}$ Whether LM and sclerosing lymphocytic lobulitis are prelymphomatous remains to be seen. In the case under discussion the presence of infiltrating carcinoma may have acted as the immunological trigger for the development of the lymphoid infiltrate. As to why uninvolved lobules should be predominantly affected is a matter for conjecture. Perhaps some ultrastructural aberration in the lobular epithelium attracted the lymphocytes. An alternative consideration is that there are two unrelated pathologies occurring in the same breast tissue. A more remote possibility is that the LM changes predisposed to the development of the lobular carcinoma, similar to the way in which Hashimoto's thyroiditis is meant to make the thyroid more susceptible to cancers.

1 Schwartz IS, Strauchen JA. Lymphocytic mastopathy. An autoimmune disease of the breast? $\mathrm{Am} \mathcal{7}$ Clin Pathol autoimmune disease

2 Mills SE. Lymphocytic mastopathy, a "new" autoimmune disease? Am $\mathcal{F}$ Clin Pathol 1990;93:834-5.

Lammie GA, Bobrow LG, Staunton MDM, Levison DA Page G, Millis RR. Sclerosing lymphocytic lobulitis of the breast-evidence for an autoimmune pathogenesis. Histopathology 1991;19:13-20.

4 Black MM, Speer FD. Periductal lymphoid infiltrations in mammary tissue. Arch Pathol 1955;60:457-61.

5 Page DL, Anderson TJ. Infiltrating carcinoma: major histological types. In: Histopathology of the breast. New York: Churchill Livingstone, 1987:193-235.

6 Hugh JC, Jackson FI, Hanson J, Poppema S. Primary breast lymphoma. An immunohistologic study of 20 breast lymphoma. An immunohistol.

7 Fishleder A, Tubbs R, Hesse B, Levine $H$. Uniform detection of immunoglobulin gene rearrangement in benign lympho-epithelial lesions. $N$ Engl f Med 1987 316:1118-21.

Figure 2 Lobulocentric lymphoid aggregates permeating lobular epithelium resulting in lymphoepithelial lesions (arrow). The infiltrate showed focal organisation into lymphoid follicles (haematoxylin and eosin). 